memo (2021) 14:7-8

https://doi.org/10.1007/s12254-021-00676-7

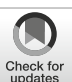

\section{Best of ASCO 2020}

\author{
Matthias Preusser
}

Accepted: 8 January 2021

(c) Springer-Verlag GmbH, AT part of Springer Nature 2021

In this issue of the Magazine of European Medical Oncology (MEMO), we provide a series of articles summarizing the most relevant presentations delivered at ASCO (American Society of Clinical Oncology) 2021 on breast cancer, non-small-cell lung cancer (NSCLC), head and neck cancer and genitourinary cancers.

Dr. Bartsch discusses the ASCO 2020 presentations in breast cancer and emphasizes results on immunotherapy with pembrolizumab in triple-negative breast cancer, and olaparib in homologous recombination deficient tumors due to genomic alterations in patients with germline PALB2 and somatic BRCA1/2 mutations. The HER2CLIMB trial established tucatinib in combination with trastuzumab and capecitabine as new standard treatment for patients with pretreated HER2-positive breast cancer brain metastases. PIK3Ca inhibition is introduced as possible therapy following CDK4/6 inhibition in hormone-receptor positive/HER2-negative breast cancer [1].

Drs. Horvath and Pircher highlights data showing an improvement of disease-free survival with postoperative osimertinib over standard chemotherapy in EGFR-mutated NSCLC and several studies reporting clinical benefit of various immunotherapeutics in lung cancer. Also, promising phase II results with the drug conjugate trastuzumab deruxtexan in HER2mutated NSCLC are discussed, thus overall underlying that substantial advances are made with modern therapies of lung cancer [2].

Dr. Füreder summarizes data on de-escalation strategies in low-risk patients and the optimal plat-

Univ.-Prof. Dr. M. Preusser $(\bowtie)$

Clinical Division of Oncology, Department of Medicine I,

Medical University of Vienna, Währinger Gürtel

18-20, 1090 Vienna, Austria

matthias.preusser@meduniwien.ac.at inum-dosing in combination with radiotherapy in high-risk patients with head and neck cancer, as well as on the role of immunotherapy in the first-line recurrent/metastatic setting [3].

Dr. Pichler and colleagues report the practicechanging data of the JAVELIN Bladder 100 trial on avelumab after response to platinum-based chemotherapy in metastatic urothelial carcinoma. In addition, they discuss the efficacy of sequential and combination treatment strategy with immune-checkpoint inhibitors and targeted agents in metastatic renal cell carcinoma. In prostate cancer, PyL-PET/CT showed promise as diagnostic tool for occult metastases and 177Lu-PSMA-617 as therapeutic tool [4].

Overall, a number of highly interesting, and in part practice-changing, data have been presented in several tumor types at ASCO 2020 and show that novel diagnostic and therapeutic approaches based on biological insights continue to contribute to significantly extend oncologists clinical management portfolio and improve outcomes of cancer patients.

Conflict of interest M. Preusser has received honoraria for lectures, consultation or advisory board participation from the following for-profit companies: Bayer, Bristol-Myers Squibb, Novartis, Gerson Lehrman Group (GLG), CMC Contrast, GlaxoSmithKline, Mundipharma, Roche, BMJ Journals, MedMedia, Astra Zeneca, AbbVie, Lilly, Medahead, Daiichi Sankyo, Sanofi, Merck Sharp \& Dome, Tocagen, Adastra. The following for-profit companies have supported clinical trials and contracted research conducted by MP with payments made to his institution: Boehringer-Ingelheim, Bristol-Myers Squibb, Roche, Daiichi Sankyo, Merck Sharp \& Dome, Novocure, GlaxoSmithKline, AbbVie.

\section{References}

1. Bartsch R. ASCO 2020: highlights in breast cancer. memo. 2021. https://doi.org/10.1007/s12254-020-00674-9. 


\section{editorial}

2. Horvath L, Pircher A. ASCO 2020 non-small lung cancer (NSCLC) personal highlights. memo. 2021. https://doi. org/10.1007/s12254-020-00673-2.

3. Füreder T. ASCO virtual meeting 2020: highlights head and neck cancer. memo. 2021. https://doi.org/10.1007/ s12254-020-00679-4.

4. Pichler R, Tulchiner G, Bektic J. Genitourinary cancers best of ASCO 2020. memo. 2021. https://doi.org/10.1007/ s12254-020-00667-0.

Publisher's Note Springer Nature remains neutral with regard to jurisdictional claims in published maps and institutional affiliations.

- For latest news from international oncology congresses see: http://www.springermedizin.at/ memo-inoncology 